\title{
Anesthesia Procedure for Congenital Insensitivity to Pain in a Child with Anhidrosis Syndrome: A Rare Case
}

\author{
Aykut Urfalioglu' ${ }^{1}$ Mahmut Arslan', Yakup Duman², Gökce Gisi', \\ Gözen Oksuz ${ }^{1}$, Hüseyin Yildiz ${ }^{1}$, Hafize Oksuz ${ }^{1}$ and Ayşe Balaban ${ }^{1}$ \\ ${ }^{1}$ Department of Anesthesia and Reanimation, Kahramanmaras Sutcu Imam University, School of Medicine, Kahramanmaras, Turkey \\ ${ }^{2}$ Department of Plastic and Reconstructive Surgery, Kahramanmaras Sutcu Imam University, School of Medicine, Kahramanmaras, Turkey
}

Congenital insensitivity to pain with anhidrosis (CIPA) syndrome is a neuropathy characterized by insensitivity to pain, impaired thermoregulation, anhidrosis, and mental retardation. A 9-year old boy with CIPA syndrome, underwent 2 operations for a calcaneal ulcer. During the first operation standard monitorization was performed. In the second operation, Bispectral Index (BIS) monitoring was added and temperature was monitored with an esophageal probe. In the first operation, in which anesthesia induction was applied with ketamine and midazolam, extremity movements with surgical stimuli were seen. Despite pain insensitivity, as extremity movements were seen with surgical stimuli, propofol was administered in the second operation. Throughout the operation, the BIS values varied from 19-58 and body temperature was measured as $36.1^{\circ} \mathrm{C}-36.9^{\circ} \mathrm{C}$. In conclusion, despite the absence of pain sensitivity in CIPA syndrome cases, there is an absolute need for the administration of anesthesia in surgical procedures because of tactile hyperesthesia.

(J Nippon Med Sch 2017; 84: 237-240)

Key words: anaesthesia, bispectral index, congenital insensitivity to pain with anhidrosis, neuropathy, thermoregulation

\section{Introduction}

Congenital insensitivity to pain with anhidrosis (CIPA) syndrome is a type 4 hereditary, sensory and autonomic (HSAN) neuropathy which is characterized by insensitivity to pain, impaired thermoregulation, anhidrosis, and mental retardation. This autosomal recessive transfer syndrome is formed through a mutation of the neurotrophic tyrosine kinase receptor (NTRK) type 1 gene, which is responsible for nerve growth factor (NGF) effects, providing development of the nociceptive, sensory and sympathetic neurons in the embryological period ${ }^{23}$.

Anhidrosis, hyperpyrexia, and febrile convulsions, which may be fatal, occur with impaired autonomic innervation of the eccrine sweat glands $s^{4,5}$, and with no sensation of pain and mental retardation, when teeth start to emerge, self-mutilation may lead to injuries of the tongue, lips, oral mucosa, eyes, fingers, and other areas. Wounds, fissures, and cavities with the addition of condi- tions such as painless fractures, neuropathic arthropathies, and chronic osteomyelitis may often require the application of orthopedic and dental surgical interventions ${ }^{6,7}$.

Early diagnosis of CIPA cases presenting to physicians with these findings, in infancy and childhood, is important in respect of both taking the necessary protective measures, and of determining the requirement for surgery. In cases where surgery is to be applied, because of the absence of pain sensation and autonomic dysfunction, there are many issues which are special features of these cases, such as the determination of the depth of anesthesia, selection of anesthesia type, the anesthetic drug, dosage, and the risk of hyperpyrexia.

\section{Case Presentation}

Informed consent was obtained from the parents of the patient for this scientific publication. A 9-year-old boy

Correspondence to Aykut Urfalioglu, MD, Kahramanmaras Sutcu Imam University, School of Medicine, Avsar Campus, Kahramanmaras, Turkey

E-mail: aykutmd1903@gmail.com

Journal Website (http://www2.nms.ac.jp/jnms/) 


\section{A. Urfalioglu, et al}

with CIPA syndrome and mental retardation, weighing $35 \mathrm{~kg}$, underwent 2 operations at an interval of 1 month for debridement and grafting of a right-sided calcaneal ulcer. The patient had widespread fissures on the skin and fingers of both hands because of self-mutilation. He had previously undergone several operations for debridement and the left leg had been amputated because of chronic osteomyelitis (Fig. 1). Laboratory tests were normal and in the first operation, monitorization was applied with standard electrocardiography (ECG), noninvasive blood pressure measurements (NIBP), oxygen saturation $\left(\mathrm{SpO}_{2}\right)$, and end-tidal $\mathrm{CO}_{2}\left(\mathrm{EtCO}_{2}\right)$. In the second operation, Bispectral Index (BIS) monitoring was added and temperature was monitored with an esophageal probe. During the preoperative intravenous (IV) cannulization, it was noticeable that the patient experienced no pain. In the first operation, anesthesia induction was applied with $50 \mathrm{mg}$ IV ketamine and $1 \mathrm{mg}$ IV midazolam and the anesthesia was maintained with $2 \%$ sevoflurane with the addition of a total of $50 \mathrm{mg}$ ketamine at intervals. Face-mask anesthesia was preferred with a 50\%/ $50 \%$ pure oxygen/air mixture and no opioids or neuromuscular blockers were used. Interestingly, throughout the surgery the patient's extremities responded to surgical stimuli, excretions were excessive on wakening but the patient was woken without any problems and was transferred to the post-anesthesia care unit (PACU). No abnormality was observed in heart rate, $\mathrm{NIBP}$ or $\mathrm{SpO}_{2}$ values.

Despite the absence of sensitivity to pain, as extremity movements were seen in response to surgical stimuli, when the second operation for the same reasons was planned, autocontrol was applied in respect of the anesthesia method to be selected, the anesthetic drugs, and the need for additional monitorization. BIS and esophageal temperature monitorization were added to the standard monitorization to be able to monitor the depth of anesthesia and body temperature. Taking target BIS values as 40-60 in the anesthesia induction using only 80 mg IV propofol and maintenance with a laryngeal mask, total intravenous anesthesia (TIVA) was applied with a propofol infusion $\left(50-100 \mathrm{mcg} / \mathrm{kg} / \mathrm{min}\right.$ ) and an $\mathrm{O}_{2} /$ air mixture (50\%/50\%).

Before induction, the BIS score was determined as 96 and during the operation it ranged from 19-58. Body temperature was measured as $36.1^{\circ} \mathrm{C}-36.9^{\circ} \mathrm{C}$. During the 35-minute operation, there were no extremity movements, hemodynamic parameters were stable and there was no requirement for opioids or neuromuscular blockers. The BIS score was checked with 5 minutes remaining of the propofol infusion, which was terminated through gradual reduction, then with removal of the laryngeal mask. When the patient recovered spontaneous respiration, and was able to raise his head (BIS score 91), he was transferred to PACU. In the unit, the patient was observed to have no pain and was transferred to the clinic after a short while. The hemodynamic parameters and BIS values of the patient in the second operation are shown in (Fig. 2).

\section{Discussion}

CIPA syndrome is a fundamental disorder of nociceptive and autonomic dysfunction formed as a result of the congenital absence of myelinated and demyelinated nerve fibers. Insensitivity to pain from birth, with increasing exposure to burns and trauma with spontaneous or selfmutilation in childhood, renders surgical intervention necessary ${ }^{8}$.

Due to the insensitivity to pain in these patients, several issues are made difficult for anesthetists in surgical procedures, such as whether or not to administer anesthesia, and if so, the choice of anesthesia type and drugs, providing sufficient depth of anesthesia, the necessity of monitoring body temperature because of impaired ther-
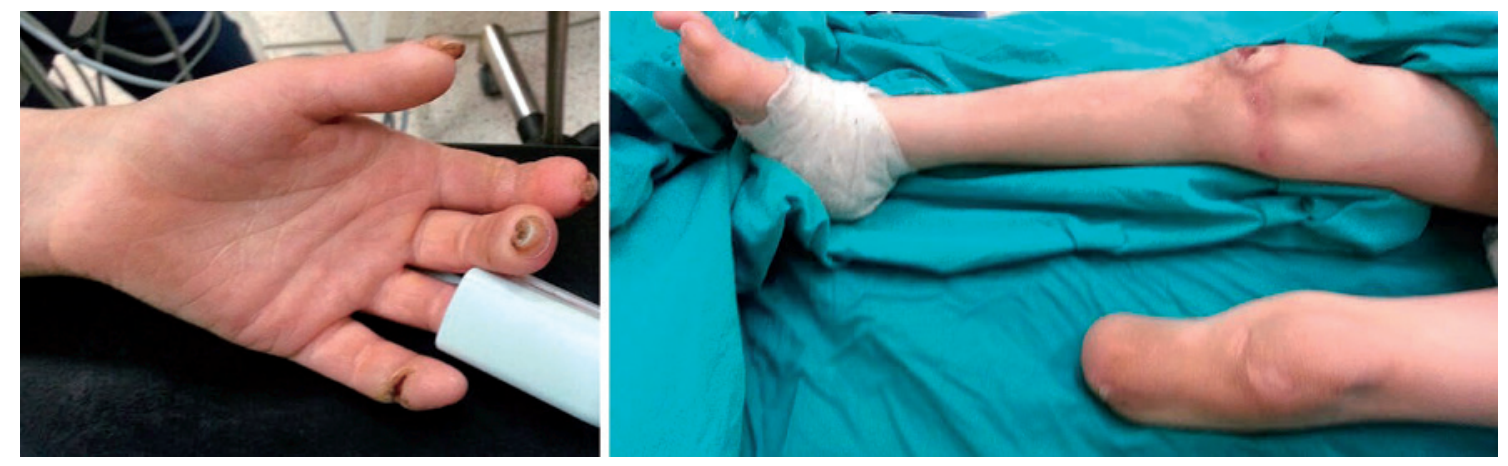

Fig. 1 


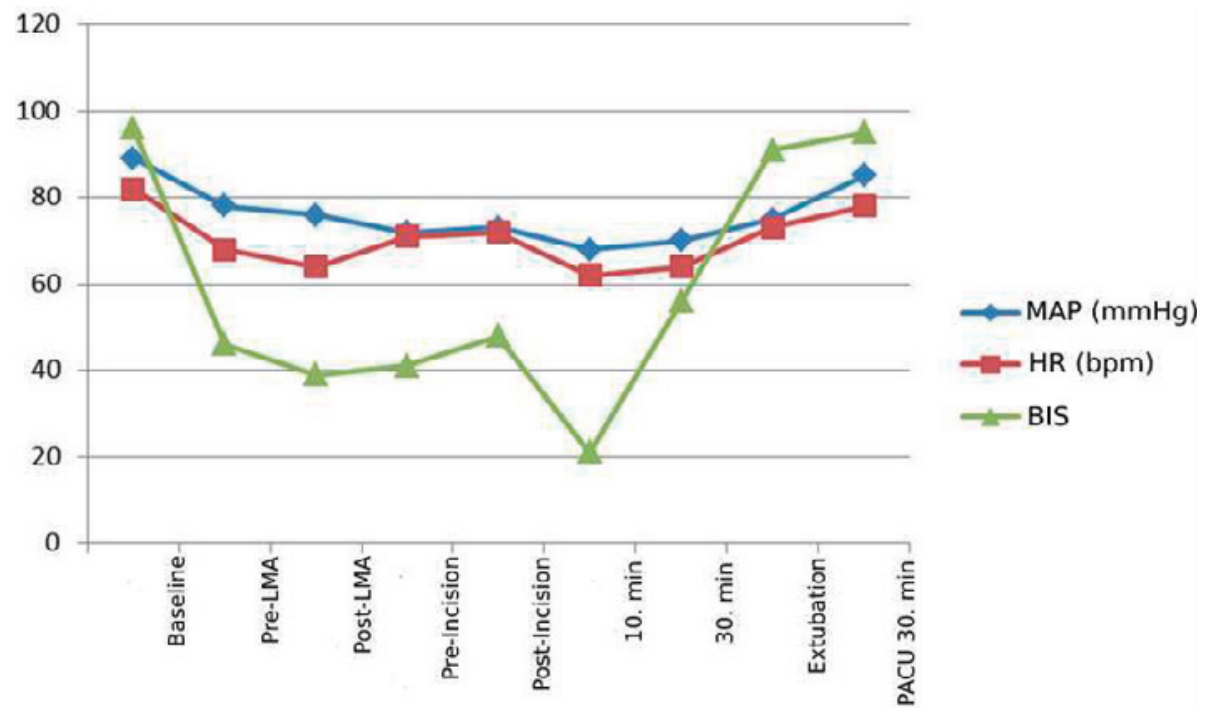

Fig. 2

(MAP: Mean Arterial Pressure, HR: Heart Rate, BIS: Bispectral Index, LMA: Larengeal Mask Airway, PACU: Post Anesthesia Care Unit)

moregulation, difficulties in providing hemodynamic control with autonomic dysfunction, and mental retardation. In early CIPA syndrome cases, it was reported that some orthopedic interventions could be made easily with sedation only because of the insensitivity to pain'. Later, with a better understanding of the clinical properties of the syndrome, and with more frequent applications of anesthesia in these cases, it was shown that during and after surgical procedures, tactile hyperesthesia could develop, which is defined as an unpleasant sensory stimulus different to the sensation of pain ${ }^{10}$. When a patient is insensitive to pain, problems can be created in the movement of extremities with tactile stimulation during manipulation, and, just as in typical pain sensation, changes can occur in the hemodynamic parameters ${ }^{10,11}$.

Therefore, there is an absolute need for the administration of anesthesia during a surgical procedure and it has been reported that the type of anesthesia should be general anesthesia as the majority of these cases are mentally retarded pediatric cases ${ }^{10,12}$. In the case reported here, it was thought that ketamine and inhalation anesthesia would be sufficient. However, difficulties were experienced during the operation as a sufficient level of anesthesia had not been provided and frequent movements of the patient's extremities were observed. Before the second operation, a thorough examination of the literature was made for the appropriate anesthesia method.

While Tamioka et al reported that all anesthetic agents could be safely used in these cases ${ }^{12}$, in another study which analyzed the anesthetic agents used in 358 proce- dures applied under general anesthesia to 35 CIPA syndrome patients, it was seen that thiopental was used in $4 \%$ of cases, ketamine in $27 \%$ and propofol in $71 \%$, and in contrast, opioids were used in $8 \%$ and muscle relaxants in $27 \%{ }^{13}$.

In the first operation on the current case, ketamine used together with inhalation agents did not provide an adequate level of anesthesia. Therefore, with the consideration of preventing tactile stimuli, propofol with TIVA application was preferred for the second operation, which was consistent with the literature. Also, differing from the first operation, BIS monitorization was used to determine the depth of anesthesia. It has been reported in the literature that by providing an adequate level of anesthesia with BIS monitorization in these patients, anesthetic awareness could be prevented ${ }^{14,15}$, and, because of the increased risk of aspiration arising from autonomic dysfunction $^{13}$, the selection of propofol rather than ketamine is thought to be more appropriate as it does not have the effect of increasing secretions and is an effective anti-emetic.

Although no serious hemodynamic disorders developed in either of the two operations on the current patient, it has been reported that there could be problems during surgery on CIPA patients because of sympathetic system dysfunction and a low level of circulating catecholamines $^{11}$. Symptoms, which may be seen, are most often hypotension and bradycardia and may range from hypertension and tachycardia to cardiac arrest. Zlotnik et al evaluated operations applied to these patients 


\section{A. Urfalioglu, et al}

over a 23-year period and reported that bradycardia was seen most often and cardiac arrest occurred in only 1 patient $^{13}$.

One of the major problems in CIPA syndrrome patients is the need to closely monitor body temperature during the operation because of the risk of hypo and hyperthermia $^{8}$. Although the development of malignant hyperthermia and values $>40^{\circ} \mathrm{C}$ have not been often observed, this subject is still noteworthy ${ }^{12,13}$. In the current case, body temperature was closely monitored and was seen to be within the normal value range.

\section{Conclusion}

Despite the absence of pain sensitivity in CIPA syndrome cases, there is an absolute need for the administration of anesthesia in surgical procedures because of tactile hyperesthesia. Propofol can be considered a good choice as it can prevent awareness by providing a sufficient level of anesthesia with BIS monitorization. It is also necessary to monitor body temperature.

Conflict of Interest: The authors declare that they have no competing interests.

\section{References}

1. Kucukdurmaz F, Imren Y, Uruc V, Sen C: Congenital insensitivity to pain with anhidrosis (CIPA) manifested with chronic osteomyelitis; A case report. J Clin Anal Med 2015; 6: 230-232.

2. van den Bosch GE, Baartmans MG, Vos P, Dokter J, White T, Tibboel D: Pain insensitivity syndrome misinterpreted as inflicted burns. Pediatrics 2014; 133: e1381-1387.

3. Perez-Lopez LM, Cabrera-Gonzales M, Gutierrez-de la Iglesia D, Ricart S, Knörr-Gimenez G: Update review and clinical presentation in congenital insensitivity to pain and anhidrosis. Case Rep Pediatr 2015; 2015: 589852.

4. Langer J, Goebel HH, Veit S: Eccrine sweat glands are not innervated in hereditary sensory neuropathy type IV. An electron-microscopic study. Acta Neuropathol 1981; 54:
199-202.

5. Rosemberg S, Marie SK, Kliemann S: Congenital insensitivity to pain with anhidrosis (Hereditary sensory and autonomic neuropathy type IV). Pediatr Neurol 1994; 11: 50-56.

6. Palit A, Inamadar AC, Pandit VS, Desai VC, Lagali SS: A child with anhidrosis, pan-analgesia and self-mutilation: a unique peripheral neuropathy. Eur J Pediatr Dermatol 2012; 22: 237-241.

7. Daneshjou K, Jafarieh H, Raaeskarami SR: Congenital insensitivity to pain and anhydrosis (CIPA) syndrome; A report of 4 cases. Iran J Pediatr 2012; 22: 412-416.

8. Yanamadala G, Ussa V, Medisetty P, Gatte B: A rare case of congenital insensitivity to pain with anhydrosis. Indian J Pain 2015; 29: 118-120.

9. Layman PR: Anaesthesia for congenital analgesia. A case report. Anaesthesia 1986; 41: 395-397.

10. Oliveira CR, Paris VC, Pereira RA, Lara FS: Anesthesia in a patient with congenital insensitivity to pain and anhidrosis. Rev Bras Anestesiol 2009; 59: 602-609.

11. Okuda K, Arai T, Miwa T, Hiroki K: Anaesthetic management of children with congenital insensitivity to pain with anhidrosis. Paediatr Anaesth 2000; 10: 545-548.

12. Tomioka T, Awaya Y, Nihei K, Sekiyama H, Sawamura S, Hanaoka K: Anesthesia for patients with congenital insensitivity to pain and anhidrosis: a questionnaire study in Japan. Anesth Analg 2002; 94: 271-274.

13. Zlotnik A, Natanel D, Kutz R, Boyko M, Brotfain E, Gruenbaum BF, Gruenbaum SE, Bodner L: Anesthetic Management of Patients with Congenital Insensitivity to Pain with Anhidrosis: A Retrospective Analysis of 358 Procedures Performed Under General Anesthesia. Anesth Analg 2015; 121: 1316-1320.

14. Matsota P, Pandazi A, Loizou M, Kostopanagiotou G: Anaesthetic management of a Greek patient with congenital insensitivity to pain and anhidrosis undergoing a scheduled orthopaedic operation. Arch Med Sci 2009; 5: 115116.

15. Brandes IF, Stuth EA: Use of BIS monitor in a child with congenital insensitivity to pain with anhidrosis. Paediatr Anaesth 2006; 16: 466-470.

(Received, November 11, 2016)

(Accepted, June 26, 2017) 